\title{
Unilateral pulmonary oedema and Pseudomonas pneumonia
}

\author{
J. UPPINGTON* \\ M.B. B.S., D.R.C.O.G., D.A., F.F.A.R.C.S. \\ M. D. Penney $\dagger$ \\ B.Sc., M.B.
}

Departments of * Anaesthetics and $\uparrow$ Pathology, Bristol Royal Infirmary, Bristol BS2 $8 H W$

\begin{abstract}
Summary
A case presented clinically as unilateral pulmonary oedema; this was subsequently revealed to be due to unilateral Pseudomonas pneumonia.
\end{abstract}

\section{Introduction}

Pulmonary oedema rarely presents unilaterally and when it does it may pose a difficult diagnostic problem. The oedema is the result of a disparity between forces governing the movement of fluid into and out of the pulmonary capillary bed, but in the case of unilateral pulmonary oedema the alteration in the balance of forces is predominantly one-sided. Unilateral pulmonary oedema has recently been reviewed (Calenoff, Kruglick and Woodruff, 1978; Leading Article, 1978), and the aetiological factors listed, but bacteriological causes were not included. A patient is described who presented clinically with unilateral pulmonary oedema and was later shown to have been suffering from unilateral Pseudomonas pneumonia. No previous reported cases of Pseudomonas pneumonia have demonstrated severe oedema.

\section{Case report}

A 52-year-old man presented to casualty in status asthmaticus. He was a known asthmatic, whose only previous admission had been for a myocardial infarction 5 years earlier. Before this admission he was not receiving any antimicrobial therapy. Initially he was treated with oxygen by mask, i.v. aminophylline and hydrocortisone but he deteriorated until he became comatose. He was transferred to the intensive care ward, intubated and mechanically ventilated. Intravenous aminophylline and hydrocortisone were continued and cloxacillin commenced. Later, the bronchodilator therapy was changed to nebulized salbutamol. Some improvement in his condition occurred over the next $24 \mathrm{hr}$, despite peristent severe bronchospasm. A chest X-ray at this time showed clear lung fields with a normal sized heart (Fig. 1). The white blood count (WBC) was $17.0 \times 10^{9} / 1$ with neutrophilia. The patient was apyrexial, alert and co-operative between periods of sedation. A mucoid trachael aspirate taken at this time showed no bacteria or pus cells on Gram staining but subsequently grew $P$. aeruginosa in culture.

Thirty-three hours after admission the patient suddenly became shocked with a BP of $60 / 0 \mathrm{mmHg}$, a pulse of $140 / \mathrm{min}$ and a temperature of $39.9^{\circ} \mathrm{C}$. Auscultation revealed bronchospasm as before but with coarse crepitations over the whole right lung. Trachael aspiration produced copious amounts of frothy blood-stained sputum. The WBC had fallen to $3.2 \times 10^{9} / 1$ with $83 \%$ neutrophils with a marked left shift. A supine chest X-ray showed complete obliteration of the right lung field, without mediastinal shift (Fig. 1). Pleural aspiration was dry. Blood and a tracheal aspirate were sent for culture and the antimicrobial therapy was changed to cefuroxine and gentamicin.

Forty hours after admission, however, the patient suffered a cardiac arrest and was unresponsive to resuscitation. Blood cultures and the tracheal aspirate subsequently grew $\boldsymbol{P}$. aeruginosa in pure culture.

At post-mortem, the right lung $(2150 \mathrm{~g})$ was grossly oedematous, overshadowing the congestion and oedema of the left lung $(920 \mathrm{~g})$. There was no free fluid present. Histologically the right lung showed focal haemorrhagic and necrotizing pneumonic consolidation with round cell and neutrophil infiltration. Numerous Gram-negative bacilli were present within the consolidated areas and were also found in the walls of necrotic arteries; however, no vascular thrombosis was seen. No pneumonic consolidation was present in the left lung. Swabs taken from the right lung tissue yielded a heavy growth of $P$. aeruginosa in pure culture.

\section{Discussion}

It is well known that Pseudomonas readily colonizes the upper respiratory tract of patients who are in hospital, who are debilitated or who are taking broad-spectrum antibiotics (Noone and 

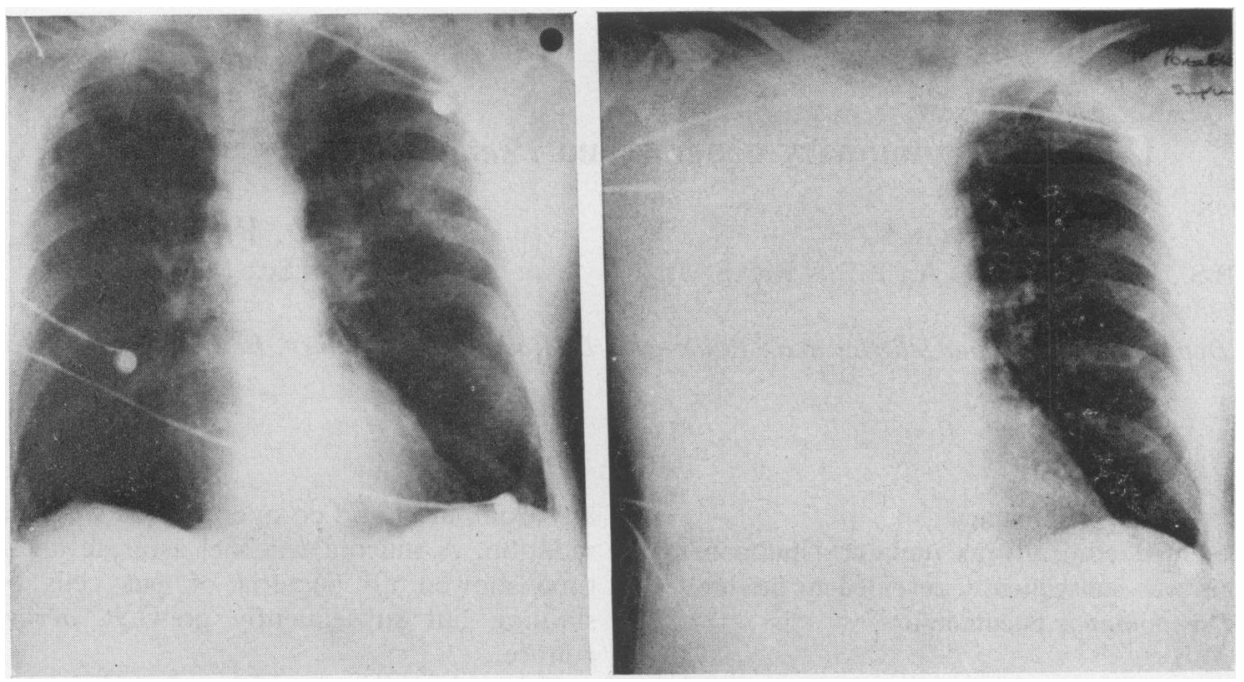

FIG. 1. (a) a chest X-ray taken on the morning after admission showing normal lung fields, (b) a chest $\mathrm{X}$-ray taken $24 \mathrm{hr}$ later showing complete obliteration of the right lung field, without mediastinal shift.

Rogers, 1976). Pseudomonas pneumonia is a recognized complication of positive pressure ventilation using reservoir nebulizers and is often associated with pre-existing lung disease. A right-sided pneumonia in this patient suggested aspiration but there was no evidence of gross contamination of the lungs with gastric contents.

Oedema is a recognized macroscopic finding in Pseudomonas pneumonia but it is usually not severe and is overshadowed by other macroscopic features, such as focal haemorrhagic or yellow lobulated areas (Fetzar, Werner and Hagstone, 1967). In a series of 10 patients with Pseudomonas pneumonia (Tillotson and Lerner, 1968), only one developed fatal pulmonary oedema, but it was not made clear whether this was directly related to the pneumonia or secondary to congestive cardiac failure. It is a recognized feature of Pseudomonas pneumonia that affected vessels rarely thrombose, as in this case, and therefore leakage of fluid may occur from the blood into the interstitium and thence into the alveolar spaces. The gross increase in capillary permeability that must have occurred in this case may have been due to ischaemia from vasculitis (Fetzar et al., 1967), local release of vasoactive substances, endotoxin, activation of complement system via Hageman factor, release of lysosomal enzymes (Clowes, 1973) or direct damage by the bacterium itself. Oedema of the lett lung compared $\frac{\mathbb{D}}{\mathbb{D}}$ to the right was insufficient for diagnosis during life 3 and was probably secondary to shunting of blood from the right to the left lung. The reason for suef $\vec{\theta}$ gross unilateral oedema in this case is not entirey ${ }^{\infty}$ clear but it did occur in direct association wif unilateral Pseudomonas pneumonia.

\section{Acknowledgments}

We thank Dr John Bowes for his encouragement and Dr M. J. Campell for permission to report details of this case.

\section{References}

Calenoff, L., Kruglick, G.D. \& Woodruff, A. (1978) 官 Unilateral pulmonary oedema. Radiology, 126, 19.

Clowes, J.R. (1973) The pulmonary response to sepsis ando endotoxin. Immuno-symposium, Vienna, Sept. 1973. Published In: Gram-Negative Bacterial Infections (Ed byं Urbaschek, B., Urbaschek, R. \& Neter, E.), p. 419.3 Springer-Verlag, Berlin.

Fetzar, A.E., Werner, A.S. \& Hagstone, J.W.C. (1967)o Pathological features of pseudomonal pneumonia.

American Review of Respiratory Disease, 96, 1121 .
LEADING ARTICLE (1978) Unilateral pulmonary oedema. British Medical Journal, 1, 1574.

NOONE, P. \& Rogers, B.T. (1976) Pneumonia caused by coliforms and Pseudomonas aeruginosa. Journal of Clinicaht Pathology, 29, 652.

Tillotson, J.R. \& LeRner, A.M. (1968) Characteristics of non-bacteraemic Pseudomonas pneumonia. Annals of Internal Medicine, 68, 295. 